\title{
EL NIVEL DE CONOCIMIENTO SOBRE SALUD SEXUAL Y \\ REPRODUCTIVA Y SU INFLUENCIA EN EL RENDIMIENTO ACADÉMICO DE LOS ALUMNOS ADOLESCENTES DEL CENTRO DE ESTUDIOS PREUNIVERSITARIOS (CEPU) 2013-I DE LA UNIVERSIDAD NACIONAL JORGE BASADRE GROHMANN DETACNA
}

\author{
THE LEVEL OF KNOWLEDGE ABOUT SEXUAL AND REPRODUCTIVE \\ HEALTH AND ITS INFLUENCE ON THE ACADEMIC PERFORMANCE \\ ADOLESCENT STUDENTS PREUNIVERSITARIOS STUDIES CENTER (CEPU) \\ 2013-I UNIVERSITY JORGE BASADRE GROHMANN NATIONAL TACNA
}

\begin{abstract}
Humberto B. Vargas Pichon'; Juan Bornáz Acosta ; Luis Solórzano Espinola ; Eduardo Rodríguez Delgado; Ita Huamán Guzman
\end{abstract}

\begin{abstract}
RESUMEN
El presente Trabajo de Investigación es un estudio explicativo que se efectuó durante el periodo Abril 2012 a Marzo 2013 en el Centro de Estudios Preuniversitarios (CEPU) de la Universidad Nacional Jorge Basadre Grohmann de Tacna $(U N J B G)$. El diseño muestral fue probabilístico, sistemático y considerando el muestreo estratificado. Se trabajó sobre una población de 1140 alumnos del Centro de Estudios Preuniversitarios (CEPU) 2013-I de la Universidad Nacional Jorge Basadre Grohmann de Tacna y con una muestra de 133. Para indagar el nivel de conocimientos sobre Salud sexual y Reproductiva se utilizó como instrumento un Inventario con preguntas referentes al tema. Por otro lado, para la determinación del Rendimiento Académico se usó la información brindada por la Oficina del CEPU de la UNJBG.Asimismo, se utilizó el Minitab 16 para el procesamiento de datos y la elaboración de los cuadros y gráficos estadisticos, lo que nos permitió obtener la conclusión principal y conclusiones específicas correspondientes.
\end{abstract}

Palabras clave: Salud sexual y reproductiva y rendimiento académico.

\section{ABSTRACT}

The present investigation is an explanatory study that was conducted during the period April 2012 to March 2013 in the Center for Pre-University (CEPU), National University of Tacna Jorge Basadre Grohmann (UNJBG). The systematic probability sample design was stratified sampling and considering we worked on a population of 1140 students and a sample of 133 students from Pre-University Studies Center (CEPU) 2013-I of the National University of Tacna Jorge Basadre Grohmann. To determine the level of knowledge about sexual and reproductive health an inventory tool was used with questions regarding the issue of sexual and reproductive health. On the other hand, for the determination of the Academic Performance was used information provided by the Office of the UNJBG CEPU. Besides the Minitab 16 for data processing and preparation of statistical charts and graphs, allowing us to obtain the main conclusion and specific conclusions corresponding conclusions.

Keywords: Sexual and reproductive health and academic performance. 


\section{I.- INTRODUCCIÓN}

En el proceso enseñanza-aprendizaje frecuentemente se percibe diferencias sobre conocimientos de Salud Sexual y Reproductiva, las cuales influyen en el aprendizaje, en el Rendimiento Académico y en el desarrollo personal de los estudiantes. tales diferencias deben ser consideradas por el profesor para adaptar sus técnicas metodológicas.

La presente investigación valora sistemáticamente el nivel de conocimientos sobre salud sexual y reproductiva y su influencia en el rendimiento académico de los alumnos adolescentes del Centro de Estudios Preuniversitarios (CEPU) 2013-I de la Universidad Nacional Jorge Basadre Grohmann de Tacna.

El resultado, con un nivel de significancia de 0,10 , indica lo siguiente: Existe una BAJA influencia $(2.7 \%)$ del nivel de conocimientos sobre Salud Sexual y Reproductiva en el Rendimiento Académico de los alumnos del Centro de Estudios Preuniversitarios (CEPU) 2013-I de la Universidad Nacional Jorge Basadre Grohmann de Tacna.

\section{II.- DESARROLLO DEL TEMA}

\subsection{Salud sexual}

La salud sexual es definida por la Organización Mundial de la Salud (OMS) como "un estado de bienestar físico, emocional, mental y social relacionado con la sexualidad; la cual no es solamente la ausencia de enfermedad, disfunción o incapacidad. Para que la salud sexual se logre y se mantenga, los derechos sexuales de todas las personas deben ser respetados, protegidos y ejercidos a plenitud".

Por su parte, la Organización Panamericana de la Salud (OPS) ha definido la salud sexual como "la experiencia del proceso permanente de consecución de bienestar físico, psicológico y sociocultural relacionado con la sexualidad."

Ambos organismos consideran que, para que pueda lograrse y mantenerse la salud sexual, deben respetarse los derechos sexuales de todas las personas. En efecto, para lograrla, la OMS asegura que se requiere un "enfoque positivo y respetuoso de la sexualidad y las relaciones sexuales, así como la posibilidad de tener relaciones sexuales placenteras y seguras, libres de coerción, discriminación y violencia."

\subsection{Salud reproductiva}

Salud reproductiva es, según la OMS, "el estado de completo bienestar físico, mental y social, en los aspectos relativos a la sexualidad y la reproducción en todas las etapas de la vida". No es por tanto una mera ausencia de enfermedades o dolencias. La salud reproductiva implica que las personas puedan tener una vida sexual segura $y$ satisfactoria, la capacidad de tener hijos y la libertad de decidir si quieren tenerlos, cuándo y con qué frecuencia (derechos reproductivos). Tanto el hombre como la mujer tienen derecho a estar informados y tener acceso a métodos de regulación de la fertilidad seguros, eficaces y asequibles así como a disponer de servicios accesibles de planificación familiar y salud pública que permitan la adecuada asistencia profesional a la mujer embarazada y permitan que el parto se produzca de forma segura y garantice el nacimiento de hijos sanos.

\subsection{Salud sexual y reproductiva}

Es conveniente diferenciar la salud sexual, enfocada a la salud en las relaciones sexuales y la salud reproductiva enfocada a la reproducción sexual y a la procreación. A pesar de las grandes diferencias existentes, debe resaltarse las iniciativas realizadas hasta la fecha. En algunos países se han llegado a implementar servicios y programas a nivel gubernamental. No obstante, este tema aún genera conflictos por los tabúes y mitos existentes, tales como que brindar educación y atención en esta área generar libertinaje y no libertad; que aumentará las relaciones coitales no protegidas y la precocidad sexual. El fenómeno puede ocasionar que este tipo de programas pierdan continuidad y respaldo político. En tal sentido, se aprecia la necesidad de crear espacios de discusión sobre el tema, siendo una cuestión clave para el diseño de políticas de juventud.

\subsection{El rendimiento académico}

El rendimiento académico se define como el nivel de logro que puede alcanzar un estudiante en el ambiente escolar en general o en una asignatura en particular. El mismo puede medirse con evaluaciones pedagógicas, entendidas éstas como "el conjunto de procedimientos que se planean y aplican dentro del proceso educativo, con el fin de obtener la información necesaria para valorar el logro, por parte de los alumnos, de los propósitos establecidos para dicho proceso" (Vega García, 1998.9). 


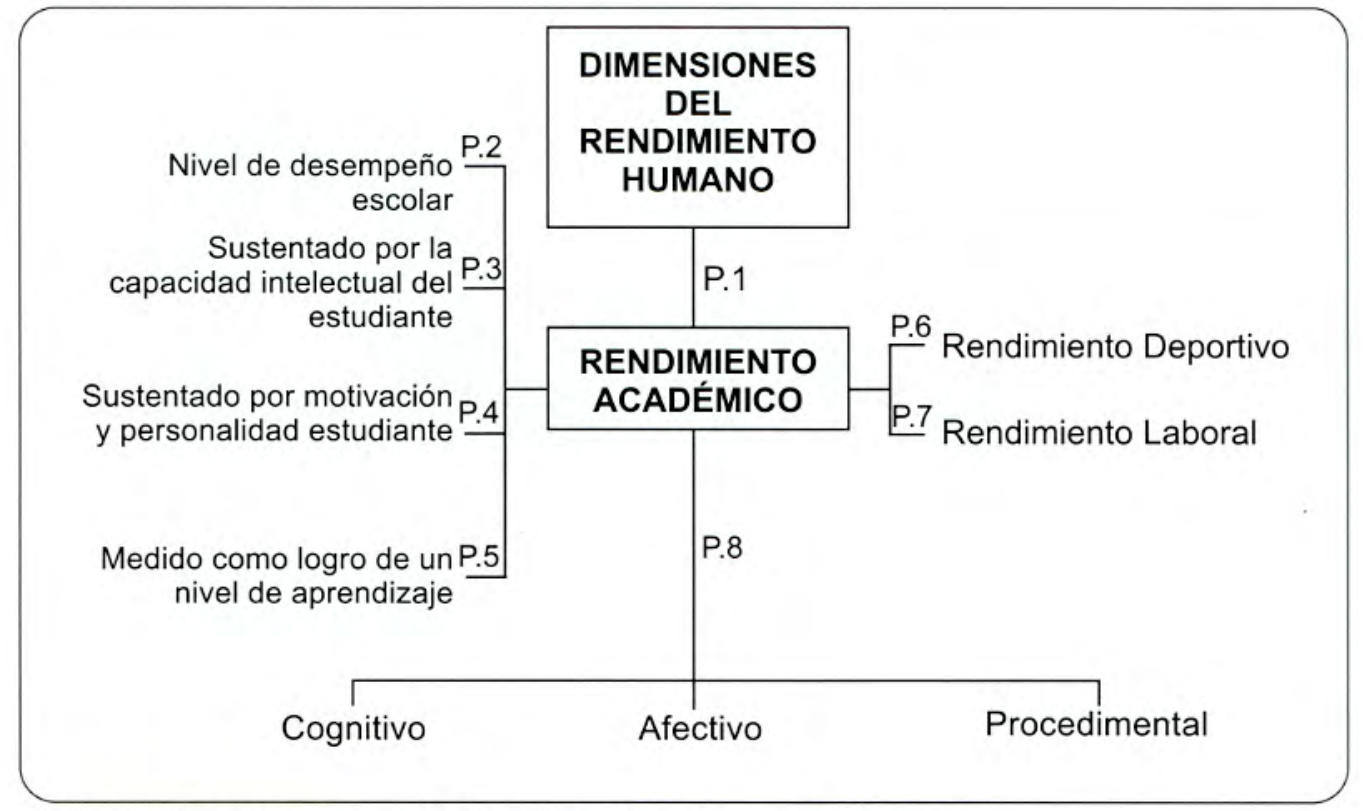

Gráfico $\mathbf{N}^{\circ} \mathbf{0 1}$. Mentefacto del Concepto "Rendimiento Académico" Fuente: http://www.psicopedagogia.com/articulos/?articulo=s11

2.5 Procesamiento y Análisis de los de datos: Usando el Software MINITAB 16 tenemos

\section{Estadísticas descriptivas: CSSSyR}

\begin{tabular}{|ccccc|}
\hline Variable & Media & Mínimo & Mediana & Máximo \\
\hline CSSSyR & 56.776 & 36.364 & 56.000 & 77.000 \\
\hline
\end{tabular}

\section{Estadísticas descriptivas: Promedio}

\begin{tabular}{|ccccc|}
\hline Variable & Media & Mínimo & Mediana & Máximo \\
\hline RA & 12.500 & 5.750 & 12.433 & 18.017 \\
\hline
\end{tabular}

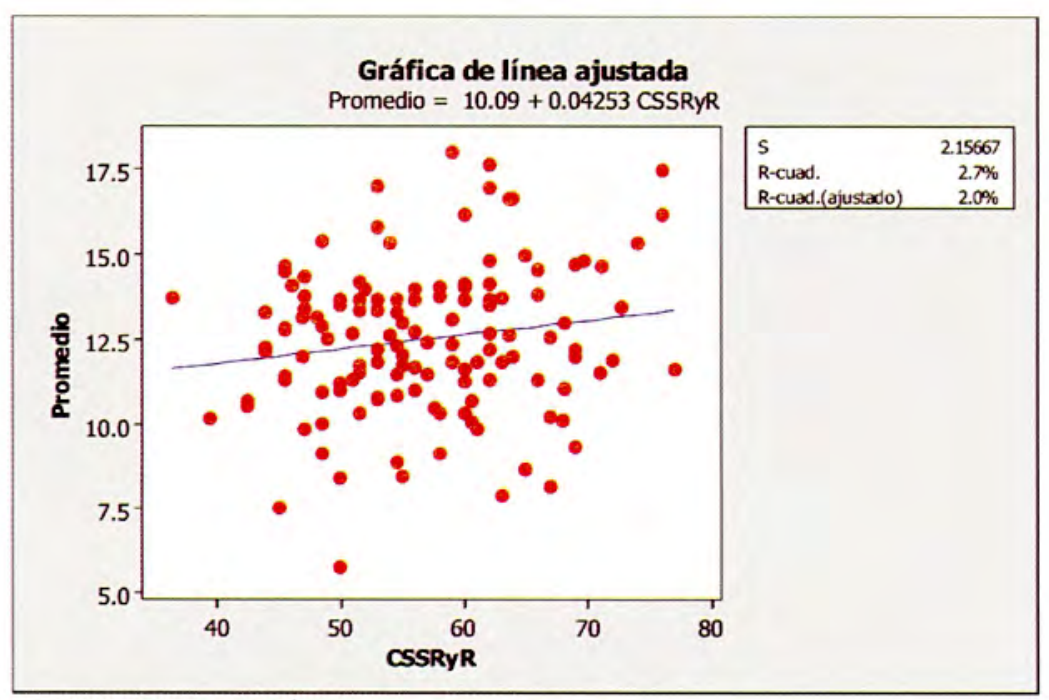

Gráfico $\mathrm{N}^{\circ}$ o2: Análisis de datos en MINITAB.

Correlación de Pearson de NCSSSR y RA $=0.163$

Valor $\mathrm{P}=0.061$ 


\section{Interpretación:}

- Teóricamente la desviación de estándar de la masa de datos es de 2.15667 .

- Teóricamente, para una puntuación de CERO (o) en nivel de conocimientos sobre salud sexual y reproductiva se incrementa en un rendimiento académico de 10.09.

- Teóricamente, existe una baja correlación $(r=$ $0.163)$ entre las variables de estudio.

- Teóricamente, el $2.7 \%$ de la variación del promedio (Rendimiento académico) se explica por la influencia de nivel de conocimientos sobre salud sexual y reproductiva. Es decir, "Existe una BAJA influencia (2.7\%) del nivel de conocimientos sobre Salud Sexual y Reproductiva en el Rendimiento Académico de los alumnos del Centro de Estudios Preuniversitarios (CEPU) 2013-I de la Universidad Nacional Jorge Basadre Grohmann de Tacna".

\subsection{Contrastación de la Hipótesis general: Hi}

El análisis del apartado anterior permite probar que "Existe una BAJA influencia $(2.7 \%)$ del nivel de conocimientos sobre Salud Sexual y Reproductiva en el Rendimiento Académico de los alumnos del Centro de Estudios Preuniversitarios (CEPU) 2013-I de la Universidad Nacional Jorge Basadre Grohmann de Tacna".

\section{III.- CONCLUSIONES}

- El promedio del Nivel de conocimientos sobre Salud Sexual y Reproductiva de la masa de datos es 56.776 (en una puntuación de 0 a 100). Por otro lado, el valor mínimo es 36.364 y el valor máximo es 77.000. Asimismo, el $50 \%$ de la masa de datos se encuentran en un nivel por encima de 56.000 (Valor de la mediana). Esto es, mas del $50 \%$ de la masa de datos esta por debajo del promedio.

- El promedio del rendimiento académico de la masa de datos es 12.500 (en una puntuación de o a 20). Por otro lado, el valor mínimo es 5.750 y el valor máximo es 18.017. Asimismo, el $50 \%$ de la masa de datos se encuentran en un nivel por encima de 12.433.

-El Nivel de Conocimientos sobre Salud Sexual y Reproductiva NO influye en forma directa y significativa en el Rendimiento Académico de los alumnos adolescentes del Centro de Estudios Preuniversitarios (CEPU) 2013-I de la Universidad Nacional Jorge Basadre Grohmann de Tacna.

\section{IV.- RECOMENDACIONES}

- Debido a que la encuesta sobre conocimientos sobre Salud Sexual y Reproductiva se aplicó en un solo momento habría que aplicarlo en dos momentos diferentes.

- Se debe implementar programas de capacitación sobre Salud Sexual y Reproductiva hacia los docentes y estudiantes de las instituciones educativas de Tacna para mejorar su nivel de conocimientos en Salud Sexual y Reproductiva.

- Se sugiere que se siga trabajando en futuras investigaciones sobre el tema y que sean del tipo experimental.

\section{REFERENCIAS BIBLIOGRÁFICAS}

\section{Fuentes Bibliográficas}

Aramburú, C. (2000). Fecundidad y planificación familiar: comparando las ENDES 2000 y 2004. Economía y Sociedad CIES 56: 19-26.

Cáceres, C. (1998). Jóvenes varones en Lima: Dilemas y estrategias en salud sexual. En: Valdés T, Olavaria J (eds). Masculinidades y equidad de género en América Latina. FLACSO - UNFPA. Santiago de Chile: FLACSO UNFPA. p. $158-74$

Coe, A. (2001). Salud, derechos y realidades de las mujeres en el Perú. Un análisis del proyecto ReproSalud. Lima: El Centro para la Salud y Equidad de Género.

Cook, R., Dickens, B. y Fathalla, M. (2003). Salud reproductiva y derechos humanos. Integración de la medicina, la ética y el derecho. Bogotá: Profamilia.

De Barbieri, T. (1994). Género y políticas de población. Una reflexión. En: Benítez R, Ramírez E (Coordinadores). Políticas de población en Centroamérica, El Caribe y México. México D.F.: UNAM/ PROLAP. p. 27794 .

De Keijzer, B. (1997). La masculinidad como factor de riesgo: masculinidad, salud mental y salud reproductiva. En: Tuñón E (ed). Género y salud en el sureste de México. Villahermosa, México: El colegio de la Frontera Sur / Universidad Juárez Autónoma de Tabasco. p. 49-70. 
Faúndes, A. y Barzelatto, J. (2005). El drama del aborto, En busca de un consenso. Bogotá: Tercer Mundo Editores.

Ferrando, D. (2000). El aborto clandestino en el Perú. Hechos y cifras. Lima: Flora Tristán / Pathfinder Internacional.

Figueroa J. (1995). Algunas reflexiones sobre la interpretación social de la participación masculina en los procesos de salud reproductiva. En: Memorias del Seminario Internacional "Fecundidad y el ciclo vital del varón en la era de la declinación de la fertilidad. Zacatecas, México: Unión Internacional para el Estudio Científico de la Población.

Gómez, E. (2002). Equidad, género y salud: retos para la acción. Rev Panam Salud Publica; 11(5): 4.54-61.

Güezmes, A., Palomino, A. y Ramos, M. (2002). Violencia sexual y física contra las mujeres en el Perú. Estudio multicéntrico de la OMS sobre la violencia de pareja y la salud de las mujeres. Lima: OMS / Centro de la Mujer Peruana Flora Tristán / Universidad Peruana Cayetano Heredia.

Güezmes, A. (2004). Reforma del sector salud y derechos sexuales y derechos reproductivos. Un enfoque integrado de derechos humanos, salud pública y género. Washington D.C.: OPS.

Hernández, J. (2006). Sexualidad masculina y reproducción. ¿Qué va decir papá?. Zacatecas, México: Coloquio Latinoamericano sobre varones, sexualidad y reproducción; 1995. Salud sexual y salud reproductiva Rev Peru Med Exp Salud Publica 23(3), 220

Lassonde, L. (1997). Los desafíos de la demografía. ¿Qué calidad de vida habrá en el siglo XXI? México D.F.: Universidad Nacional Autónoma de México.

NÚÑEZ, José y et al (1998). Estrategias De Aprendizaje, Autoconcepto Y Rendimiento Académico. En Psicothema Vol. 10, n ${ }^{\circ} 1$, pp. 97109. Colegio Oficial de Psicólogos de España.

Organización Panamericana de la Salud. (1995). Programa de mujer, salud y desarrollo. Conceptualización de género para la planificación en salud. Washington D.C.: OPS.

Palomino N, Ramos M, Valverde R, Vásquez E. Entre el placer y la obligación. Derechos sexuales y derechos reproductivos de mujeres y varones en Huamanga y Lima. Lima: FASPAUPCH / Population Concern; 2003.

Perú, Ministerio de Salud (1985). Ley $\mathrm{N}^{\circ} 26530$ que modifica el Artículo VI del Título Preliminar del Decreto Legislativo N. 346, Ley de Política Nacional de Población de 1985. Lima: MINSA.

Perú, Ministerio de Salud. (1996). Programa de salud reproductiva y planificación familiar 19962000. Lima: MINSA. Aprobado por Resolución Ministerial $N^{\circ}$ 071-96-SA/DM.

Perú, Ministerio de Salud (1999). Normas del Programa de Planificación Familiar. Resolución Ministerial N ${ }^{\circ}$ 465-99-SADM. Lima: MINSA.

Perú, Ministerio de Salud. (2004). Análisis de la situación de salud del Perú 2003. Lima: MINSA.

Quintana, A. y Vásquez, E. (1999). Construcción social de la sexualidad adolescente. Género y salud sexual. Lima: Instituto de Educación y Salud.

Ramos, M., Chirinos, J. y Vásquez, E. (2000). Los hombres y la salud sexual y reproductiva: perspectiva de los hombres y de los proveedores de servicios. Identificación de necesidades. Lima: UPCH / UNFPA / MINSA. [Inédito]

Ramos, M., Chirinos, J, y Vásquez, E. (2004). Servicios de salud sexual y reproductiva para varones desde la mirada masculina. En: Álvarez L.

Rodríguez A (coord.). La Investigación en salud Sexual y reproductiva. Propuestas metodológicas y experiencias. La Habana, Cuba: Publicaciones Azucareras. p. 94-113.

Ramos, M. (2003). Salud mental y violencia estructural en varones de sectores urbanos pobres. En: Cáceres C, Cueto M, Ramos M, Vallenas S (coord). La salud como derecho ciudadano. Perspectivas y propuestas desde América Latina. Lima: Universidad Peruana Cayetano Heredia. p. 309-18.

Ramos, M. (2003). ¿Qué pensaban los hombres antes del proceso educativo? En: Keijzer B, Ramos M, Viviano T. Abriendo nuestros ojos. Una experiencia de trabajo con hombres en temas de género y salud sexual y reproductiva. Lima. Manuela Ramos / REPROSALUD. p. 3554. 
Ramos, M. (2002). Sistematización de los talleres educativos con varones sobre salud sexual y reproductiva. Lima: REPROSALUD / Manuela Ramos. [Inédito]

SALAS, R. (1994). Hacia una metodología pluridimensional o multivariada a través de los estilos cognoscitivos de aprendizaje, Revista Estudios pedagógicos $\mathrm{N}^{\mathrm{o}}$ 20: 35-50, Valparaíso.

Saravia, C. y et al (1999). Itinerario del acceso al condón en adolescentes de Lima, Cusco e Iquitos. En: Cáceres C (editor). Investigaciones recientes sobre salud sexual y reproductiva de los jóvenes en el Perú. Nuevos retos”. Lima: REDESS Jóvenes; 1999. p. 95-116.

Távara, L. (2003). La situación de la salud reproductiva en el Perú: Aún hay motivo de preocupación. Maryland: El Centro para la Salud y Equidad de Género.

Viveros M. (1998). Decisiones reproductivas y dinámicas conyugales, el caso de la elección de la esterilización masculina. En: Valdés T, Olavaria J. Masculinidades y equidad de género en América Latina. Santiago de Chile: FLACSO UNFPA.

Yon, C. (2001). Hablan las mujeres andinas. Preferencias reproductivas y anticoncepción. Lima: Movimiento Manuela Ramos.

\section{Fuentes Hemerográficas}

Ley No 27397 (2000) Códigos de los niños y adolescentes Perú

Llosa, C. (2008). Conocimientos sobre Sexualidad y Factores que intervienen en el Inicio de Prácticas Sexuales en Estudiantes Universitarios del Primer Año de las Facultades de Ciencias Médicas, Obstetricia y Enfermería en la UNJBG de Tacna, 2008. Revista Ciencia \& Desarrollo de la Universidad Nacional Jorge Basadre Grohmann de Tacna, 12, 7-10.

Ministerio de Educación: Programa Nacional de Educación Familiar y Sexual. Guía de Educación Familiar y Sexual. Bogotá: Profamilia; 2003.

Prospecto del Proceso de Admisión 2013, Universidad Nacional Jorge Basadre Grohmann de Tacna.

\section{Fuentes Electrónicas}

Conocimientos y actitudes sobre sexualidad de los adolescentes de nuestro entorno.

Barella Balboa JL 1, Mesa Gallardo I 2, Cobeña Manzorro M 8. 1 Médico general. Centro de Salud de Úbeda (Jaén), 2 Especialista en Medicina Familiar y Comunitaria. Centro de Salud de Beas de Segura (Jaén), 3 Especialista en Medicina Familiar y Comunitaria.

Disponible en URL:

http://www.samfyc.es/Revista/PDF/v3n4/06 .pdf [Consulta 04 de enero del 2012]

Mejía O., Luz Mery;Ortiz Z., Ana Cecilia Comprensión de la salud sexual y reproductiva de los adolescentes en el marco del desarrollo a escala humanaRevista de la Facultad Nacional de Salud Pública, Vol. 24, Núm. 1, enero-junio, 2006, pp. 40-47

Disponible en URL:

http://www.samfyc.es/Revista/PDF/v3n4/o6 .pdf [Consulta 04 de enero del 2012]

La salud sexual y la salud reproductiva desde la perspectiva de género

Miguel Ramos Padilla*

Disponible en URL:

http://www.scielo.org.pe/pdf/rins/v23n3/a 10 v23n3.pdf [Consulta 04 de enero del 2012]

Informe de la estrategia sanitaria de salud sexual y reproductiva

Responsable: Lic. Obst. Gloria Emperatriz

Monroy Cárdenas

Tacna, Octubre 2009

Disponible en

URL:http://www.tacna.minsa.gob.pe/uploads /desp/Informe_ESSSR_3erTrim09.pdf

[Consulta 27 de marzo del 2012]

\section{Correspondencia:}

Humberto B. Vargas Pichón

Ciudad Universitaria fundo "Los Granados"

Av. Miraflores s/n Tacna - Perú

Juan Bornaz Acosta

Ciudad Universitaria fundo "Los Granados" Av. Miraflores s/n Tacna - Perú

Luis Solorzano Espinola

Ciudad Universitaria fundo "Los Granados" Av. Miraflores s/n Tacna - Perú

Eduardo Rodriguez Delgado

Ciudad Universitaria fundo "Los Granados"

Av. Miraflores s/n Tacna - Perú

Ita Daneri Huaman Guzman

Ciudad Universitaria fundo "Los Granados"

Av. Miraflores s/n Tacna - Perú 